\title{
Treatment of Diabetic Retinopathy (DR) and Diabetic Macular Edema (DME)
}

\author{
George Chiou CY* \\ ${ }^{1}$ Texas A\&M University Health Science Center Houston,USA \\ ${ }^{2}$ Director of New Drug R\&D CenterNatureWise Biotech and Medicals Corp, Taiwan
}

Submission: June 26, 2018; Published: July 16, 2018

*Corresponding author: George Chiou CY, Texas A\&M University Health Science CenterHouston, USA, Director of New Drug R\&D Center Nature Wise Biotech and Medicals Corp, Taiwan Email: Chiou@medicine.tamhsc.edu

\section{Introduction}

The worldwide prevalence of diabetic retinopathy (DR) is at a staggeringly high level, $127 \mathrm{M}$ patients in 2010 and will grow to $191 \mathrm{M}$ by 2030. It is a leading cause of blindness in workingage populations [1]. Obviously, an ideal potent and safe drug is urgently needed for its treatment. However, none is available despite utmost efforts have been made by numerous research institutions. Current treatment of DR includes anti-VEGF for angiogenesis suppression; intravitreal steroid injection for inflammation inhibition; laser surgery for stopping vascular leakage; and vitrectomy for hypoxia [2-5]. None of these treatments are ideal as they are invasive in delivery and disliked by patients. Besides, VEGF is an essential ingredient for normal formation and function of blood vessels and thus a complete elimination with VEGF inhibitors could cause serious adverse effects, whereas steroid injection causes systemic side effects which are resisted by both patients and doctors. Thus, even though various means are available, there is only one, VEGF inhibitors, that is preferentially used at the present time.

Major efforts have been made to R\&D an ideal drug for the treatment of DR and DME without satisfactory result. The key problem probably lies on the complicated pathological etiology of DR and DME, which cannot be treated successfully with an agent aiming at eliminating only a single factor among many. It resulted in drugs which can produce only partial but not full efficacy. Therefore, a new ideal drug ought to be able to eliminate most, if not all of etiology factors simultaneously. The multiple etiology factors of DR and DME include, but not limited to formation of AGE (advanced glycation end products) via oxidative stress under hyperglycemia; subclinical inflammation; hypoxia; anoxia; induction of VEGF; over production of GFAP (glial fibrillary acidic protein); angiogenesis; vascular leakage; and the like [6]. There are more than a dozen research institutes engage in the R\&D of new drugs for the treatment of DR and DME. They are at a different stages of development between preclinical studies to phase III. All of them are aiming to suppress only part of multiple etiology factors primarily[7]. Since none is trying to suppress the whole complex etiology, the possibility of resulting in a high potent efficacious drug is quite unlikely in the near future.

\section{Background}

Osthole is a natural product isolated from Chinese herb, Cinidummonnieri(L.) Cusson. It produces potent antiinflammatory action on uveitis induced by lens protein, endotoxin, and/or interleukin-1 $[8,9]$. Because of its potent antiinflammatory action on uveitis induced by various inflammatory factors, it was selected as a prototype compound to develop various analogs of derivatives to produce anti-DR and DME actions. Among numerous derivatives synthesized, BMX turned out to be the most unique and potent agent for treating DR and DME as it produces multiple action mechanisms, including, but not limited to anti-inflammation, anti-oxidation, VEGF inhibition, blockade of vascular leakage of BRB (blood retinal barrier), antihypoxia, anti-anoxia, GFAP suppression, angiogenesis inhibition, HDAC8 (histone deacetylase isozyme \#8), etc. Because of its multiple action mechanisms to take care of the complex etiology of DR and DME, BMX could become a promising agent for the treatment of DR and DME.

\section{Preclinical Studies}

BMX is quite nontoxic in cell culture incubation. It showed cytotoxicity on RPE (retina pigment epithelium) and HVEC (human vascular epithelium cell) cultures only at the maximum concentration $(10 \mathrm{ug} / \mathrm{ml})$ studied. It reversed the cytotoxicity of HUVEC induced by $\mathrm{NaIO} 3$ (sodium iodate) at $300 \mathrm{ug} / \mathrm{ml}$ efficiently at all concentration levels tested from $0.03 \mathrm{ug} / \mathrm{ml}$ to $1 \mathrm{ug} / \mathrm{ml}$. As for oxidative stress caused by $\mathrm{H} 2 \mathrm{O} 2$ (at $400 \mathrm{uM}$ to $600 \mathrm{uM})$, it is much more efficacious to produce anti-oxidation on RPE cells than on HVEC cells at $1 \mathrm{ug} / \mathrm{ml}$ to $10 \mathrm{ug} / \mathrm{ml}$. Similar results were obtained by anti-oxidation of BMX on oxidative injury caused by tBHP. Regarding anoxia, BMX was efficacious to reverse NaN3 induced anoxia (at $3 \mathrm{mM}$ ) on RPE cells at $0.03 \mathrm{ug} /$ $\mathrm{ml}$ to $10 \mathrm{ug} / \mathrm{ml}$. 
Diabetes mellitus causes DR, a vascular complication that frequently is diagnosed and treated later than it should. DR destroys vision via retinal neovascularization and macular edema. The pathophysiology of DME involves dilated capillaries, retinal microaneurysms, and loss of pericytes with eventual impairment of the BRB (blood retinal barrier). Breakdown of the BRB results in fluid leakage into the extracellular space which disrupts macular structure and function on a cellular level. In Evans Blue leakage experiment of DRE, 1\% BMX blocked it completely and 0.5\% BMX partially, showing a good dose response relationship. GFAP (glial fibrillary acidic protein) is an established indicator of retinal stress. In the normal mammalian retina, GFAP is marginally detectable in Muller cells. When under stress, activated Muller cells express high levels of GFAP. In the present research, increased GFAP expression was demonstrated in Muller cells by Western Blot, indicating that Muller cell dysfunction was involved in STZ (streptozotocin)induced DR. Seven weeks after intraperitoneal injection of STZ, the expression of GFAP in retina of diabetic control group was significantly increased as compared with non-diabetic group $(p<0.05)$. The expression of GFAP in retina was markedly suppressed as compared with diabetic control group $(\mathrm{p}<0.05)$ after diabetic rats were instilled with 1\% and 0.5\% BMX tid for 6 weeks. The gene expression of GFAP was detected by quantitative real-time PCR as well. Similarly, the GFAP expression of diabetes retina was significantly down-regulated by treatment with $0.5 \%$ and $1 \%$ BMX in a dose dependent manner as compared with diabetic control group.

VEGF is a major regulator of blood vessel formation and function. It controls several processes in endothelial cells, such as proliferation, survival and migration. Retinal VEGF expression is correlated with diabetic BRB breakdown and ischemia related neovascularization in animals and humans. In this study, VEGF expression in Muller cells was significantly upregulated in diabetic retina, indicating the VEGF overexpression plays a crucial role in retinal vascular abnormality in STZinduced diabetes. The VEGF level in diabetic eyes was markedly suppressed by $0.5 \%$ and $1 \%$ BMX, indicating that DR and DME can be treated effectively by BMX in a dose dependent manner.

\section{Conclusion}

It has been recognized that currently used drugs and medical devises for the treatment of DR and DME are not ideal as the efficacy is not at the upmost level and the delivery/ administration is invasive with serious side effects. The key problem could be due to each medication attacks only a single and/or small part of pathogenic etiology of the diseases rather than the total complex etiology factors at the same time. A promising new novel agent could be invented, which produces anti-DR and DME through anti-inflammation, anti-oxidation, VEGF inhibition, GFAP suppression, vascular leakage blockage, anti-anoxia, anti-hypoxia, HDAC8 inhibition, anti-angiogenesis, etc. simultaneously. Thus, it could become a potent agent to treat DR and DME. Further, no major side effect has been found, if any and it could be administered orally and/or locally as eye drops.

\section{References}

1. Zheng YF, He MG, Congdon N (2012) The worldwide epidemic of diabetic retinopathy. Indian J Ophthalmol 60(5): 428-431.

2. Starita C, Patel M, Katz B, Adamis A (2007) VEGF and the potential therapeutic use of pegaptanib (Macugen) in DR. Diabetic Retinopathy. In: Lang GE, Basel (Eds.), Karger, Basel, Switzerland, pp. 122-148.

3. Jonas JB (2007) Intravitreal triamcinolone acetonide for DR. Diabetic Retinopathy. Ophthalmol 39: 96-110.

4. Lang GE (2007) Laser treatment of DR. Diabetic Retinopathy, Krager, Basel, Switzerland, pp. 48-68.

5. Wolfensberger TJ, Gregor ZJ (2010) Macular Edema-rationale for therapy. Coscas, G (Eds.), Macular Edema, Krager, Basel, Switzerland, pp. 49-58.

6. Augustin A, Loewenstein A, Kuppermann BD (2010) General pathophysiology. In: Coscas, G (Eds.), Macular Edema, Krager, Basel, Switzerland, pp. 10-26.

7. Vision Gain (2014-2024) Diabetic Retinopathy Segment.

8. Chiou GCY, Yao QS, Li BHP (1993) Inhibition of lens protein-, endotoxin-, and interleukin-1-induced inflammation with osthole and some other non-steroidal anti-inflammatory agent. Inflammo Pharmacol 2: 5-13.

9. Yang YC, Chen CN, Wu CI, Huang WJ, Kuo TY, et al. (2013) NBM-T-LBMX-OS01, semisynthesized from Osthole, is a novel inhibitor of HDAC and enhances learning and memory in rats. Evidence Based Complementary and Alternative Medicine 2013: 514908. 